\title{
Nanomedicines for Cancer Therapy: An Update of FDA Approved and Those under Various Stages of Development
}

\author{
Gopalakrishna Pillai*
}

Professor, Sullivan University College of Pharmacy, 2100 Gardiner Lane, Louisville KY 40205, USA

Received: May 18, 2014, Accepted: June 17, 2014, Published: June 27, 2014

*Corresponding author: Gopalakrishna Pillai, PhD, Professor, Sullivan University College of Pharmacy, 2100 Gardiner Lane, Louisville KY 40205, USA, Tel.: 1502413 8951; Fax.: 1502515 4669, Email: gpillai@sullivan.edu

\begin{abstract}
Nanomaterials, with their size range $(1-100 \mathrm{~nm})$ that corresponds to basic biological materials such as DNA, vastly increased surface area $\left(1000 \mathrm{~m}^{2} / \mathrm{g}\right)$ and unique mechanical, electronic, photonic and magnetic properties are projected to have a wide range of applications from drug and gene delivery to biomedical imaging and more recently to personalized medicine. The major advantages of using nanomaterials as a carrier for anticancer agents are the possibility of targeted delivery to the tumor, tumor imaging, their ability to hold thousands of molecules of a drug and also their ability to overcome solubility, stability and resistance issues. Currently, there are several nanotechnology-enabled diagnostic and therapeutic agents undergoing clinical trials and a few are already approved by the FDA. Targeted delivery of anticancer agents is achieved by exploiting a unique characteristic of the tumor cells called "the Enhanced Permeation and Retention Effect (EPR effect). In addition to this passive targeting based mainly on size, the nanoparticle surface may be modified with a variety of ligands that would interact with specific receptors over-expressed on the surface of the tumor cells, thus imparting specificity for active targeting. Site-specific release of a drug contained in a nanoparticulate system by the application of external stimuli such as hyperthermia to a thermosensitive device is another innovative strategy for targeted delivery. This review provides an update on the FDA approved cancer nanomedicines such as Abraxane, Doxil, daunoxome, Oncaspar, DepoCyt and those in nanoplatforms that have reached an advanced stage of clinical development utilizing liposomes, lipid nanoparticles, lipoplexes, albumin nanospheres, thermosensitive devices, micelles and gold nanoparticles.
\end{abstract}

Keywords: Cancer nanomedicines; Approved products and in clinical development; Targeted delivery; Stimuli-Sensitive Release; Liposomes; Albumin-bound nanoparticles; Gold nanoparticles; RNA interference

\section{Introduction}

One of the draw backs of conventional chemotherapy for the treatment of cancer is the inability to deliver the drug in adequate quantity to the tumor site without undesirable side effects. Nanomaterials, with their size range $(1-100 \mathrm{~nm})$ that corresponds to basic biological materials such as DNA, vastly increased surface area $\left(1000 \mathrm{~m}^{2} / \mathrm{g}\right)$ and unique mechanical, electronic, photonic and magnetic properties are projected to have a wide range of applications from drug and gene delivery to biomedical imaging. The major advantage of using nanomaterials as a carrier for anticancer drugs is the possibility of targeted delivery to the tumor, their ability to enclose or bind thousands of molecules of a drug and deliver to the required site and to overcome solubility and stability issues. ${ }^{[1-4]}$ In addition, nanoplatforms like liposomes, lipid nanoparticles, dendrimers, micelles, gold nanoparticles can offer solutions to various problems in diagnosis and treatment of cancer. Multifunctional nanoparticles (MNPs) for simultaneous diagnosis, imaging and treatment, an area referred to as Theranostics, are also being developed for personalized medicine..$^{[5-8]}$

Nanoparticles (NPs) loaded with chemotherapeutic agents such as the liposomes, polymer nps and lipid nps have the ability to overcome drug resistance. Multidrug resistance (MDR) is one of the biggest challenges in cancer chemotherapy. Development of cancer drug resistance is attributed to a number of factors including inefficient drug delivery to tumor cells, partly due to inefficient targeting and the rapid removal of the drug from tumor cells by the efflux pump, P-glycoprotein (P-gp). This membrane transporter is known to be over-expressed in tumors compared to drug-sensitive parent cell lines. Approximately 50\% of the anticancer drugs used clinically today are substrates of P-gp. Therefore, a plausible way to reduce MDR is to increase the efficiency of drug delivery to the tumor and reduction of MDRbased drug efflux. It has been suggested that nps may be able to circumvent P-gp-mediated resistance by partially bypassing the efflux pump as they are internalized by endocytosis..$^{[9-13]}$

The size range of nanomaterials is strictly defined as 10$100 \mathrm{~nm}$, although many marketed nanomedicines are in the submicron range of 100-1000 nm. ${ }^{[14]}$ Many of the properties of nanomaterials are different from those of the bulk materials due to an increased surface area and quantum effects. The quantum effects coupled with surface area effects can impact on the size-dependent properties of nanomaterials, which in turn can influence their in vivo behavior. Nanoparticles therapeutics is especially useful for cancer and disorders of the central nervous system, because they are able to cross the blood brain barrier (BBB). ${ }^{[15]}$ In addition, it is also possible to achieve a prolonged residence time and a desired drug-release pattern by controlling the size and architecture of nanoparticles. 


\section{Passive targeting}

Passive targeting of drug-loaded nanocarriers and their accumulation in tumor tissue is a size-dependent process. Passive targeting occurs as a result of the pathophysiological characteristics of the tumor vessels such as a leaky vasculature and poor lymphatic drainage.

The extravasation of particulate materials in to the tumor tissue and their retention is called Enhanced Permeation and Retention (EPR) effect. However, these particles, recognized as foreign bodies, may be opsonized by the cells of the reticuloendothelial system (Mononuclear Phagocyte System, MPS), thereby reducing the availability of the drug at the required site. Well-designed nanocarriers such as those coated with polyethylene glycol, a process called pegylation, have the ability to escape capture by the MPS. Such drug delivery systems are referred to as the stealth systems. ${ }^{[16]}$ Most passive- targeting nanosystems have a surface coated with PEG for biocompatibility and "stealth" purposes. A variety of pegs with varying chain length and molecular weight are being used to control the thickness of the PEG coating and the grafting efficiency. Longer chains offer greater steric influence around the nanocarrier than short chain pegs. ${ }^{[17]}$ Surface modification of nanocarriers can also be achieved by using derivatives of PEG such as the block copolymers of the poloxamer type. ${ }^{[18]}$ Genexol-PM, a micellar formulation formed from PEG-based block copolymers is an example of such a product available in the market (Genexol-PM was approved in 2007 in Korea and marketed in Europe).

\section{Active drug targeting}

Passive targeting strategies have shown several limitations. Therefore, considerable effort is underway to maximize the accumulation of nanoparticles at the site of interest by other methods. Site- specific targeting and entry in to the tumor cell can be achieved via the over-expressed receptors of the tumor cell membrane and phagocytosis/endocytosis mechanisms. ${ }^{[19]}$ The transferrin receptor, ${ }^{[20-23]}$ and the folic acid receptor over-expressed in many types of cancers are targets for active drug delivery. ${ }^{[24,25]}$ Transferrin is a cell membrane- associated glycoprotein involved in the cellular uptake of iron and in the regulation of cell growth. Iron uptake occurs via internalization of iron-loaded transferrin mediated by the transferrin receptor. Similarly, the folic acid receptor is also a useful target for tumor specific drug delivery. It is known that the folate receptor density increases as the grade of cancer worsens. Active targeting is also possible by targeting biomarkers specific to a tumor or by exploiting processes that are essential for tumor development such as neoangiogenesis. CA-125 is such a biomarker for ovarian cancer and is expressed in more than $85 \%$ cases of ovarian cancer. ${ }^{[26,27]}$ Although the benefit of active targeting is wellknown, this technology has resulted in only a few clinically validated nanoproducts to date.

Examples of Nanomedicines for Cancer Approved by FDA and Those Undergoing Clinical Trials ${ }^{28}$ (Table 1 and Figure 1).

\section{Doxil (Liposomal Doxorubicin)}

Doxil (pegylated liposomal doxorubicin), is the first
FDA approved (1995) nanodrug used to treat some types of cancers, including metastatic ovarian cancer and AIDS-related Kaposi's sarcoma. The product was formulated to improve the balance between the efficacy and toxicity of doxorubicin therapy. It contains doxorubicin (Adriamycin), a member of the anthracycline group, enclosed in an $80-90 \mathrm{~nm}$ size unilamellar liposome coated with PEG that allows the drug to stay in the bloodstream longer so that more of the drug reaches the cancer cell. $^{[28-30]}$ Doxorubicin is believed to act on cancer cells by two different mechanisms: intercalation into DNA and disruption of topoisomerase II-mediated DNA repair and generation of free radicals that result in damage to cellular membranes, DNA and proteins. ${ }^{[31]}$ Doxorubicin is known to cause severe and possibly life-threatening heart problems (eg, heart failure). These problems may occur during therapy or sometimes months to years after receiving doxorubicin. In some cases, heart problems are irreversible. Myocardial damage may lead to congestive heart failure and may occur as the total cumulative dose of doxorubicin hcl approaches $550 \mathrm{mg} / \mathrm{m}^{2}$. The risk may be increased in patient using certain medicines that may affect heart function or have a history of heart problems, receiving radiation treatment to the chest area, or previous therapy with other anthracyclines (eg, epirubicin) or cyclophosphamide.<smiles>COc1cccc2c1C(=O)c1c(O)c3c(c(O)c1C2=O)C[C@@](O)(C(=O)CO)C[C@@H]3O[C@H]1C[C@H](N)[C@@H](O)[C@H](C)O1</smiles>

\section{Doxorubicin}

The risk of developing heart problems varies depending on the dose and condition. Given as liposomes, it has fewer side effects on healthy cells than regular doxorubicin. ${ }^{[32-34]}$ Liposomal doxorubicin is also called Doxil (Johnson \& Johnson, USA), Caelyx (Janssen-Cilag, Europe), Evacet(Liposome company INC.,) and lipodox (Sun Pharma). Loading of Doxorubicin (DXR) in liposomes is achieved by an ammonium ion gradient method where the neutral form of DXR from a sucrose medium crosses the lipid bilayer and the protonated DXR formed inside the aqueous compartment with ammonium sulphate is retained. It is estimated that a liposome contains 10-15 thousand molecules of doxorubicin. Liposomes are sterically stabilized by PEG surface coating (Figure 1). This is achieved by Distearoyl-phosphatidylethanolamine-PEG (DSPE-PEG) anchored into the phospholipid bilayers made of hydrogenated soy phosphatidylcholine and cholesterol. ${ }^{[35]}$ The drug to lipid ratio is 0.125 . The use of such stealth liposomes resulted in enhanced circulation time after intravenous administration (mean residence time of 4 days) and improved the pharmacokinetic profile of the drug. The area under the curve (AUC) was $900 \mu \mathrm{g} . \mathrm{h} / \mathrm{ml}$ for Caelyx compared to $45 \mu \mathrm{g} . \mathrm{h} / \mathrm{ml}$ for the uncoated liposomes, Myocet, and $4 \mu \mathrm{g} . \mathrm{h} / \mathrm{ml}$ for doxorubicin in patients with solid tumors. ${ }^{[36,37]}$ 
Table 1: Examples of Nanomedicines for cancer approved by FDA and those undergoing clinical trials. (Source: FDA website, original publications, reviews and websites of pharmaceutical companies supplying/developing these drugs).

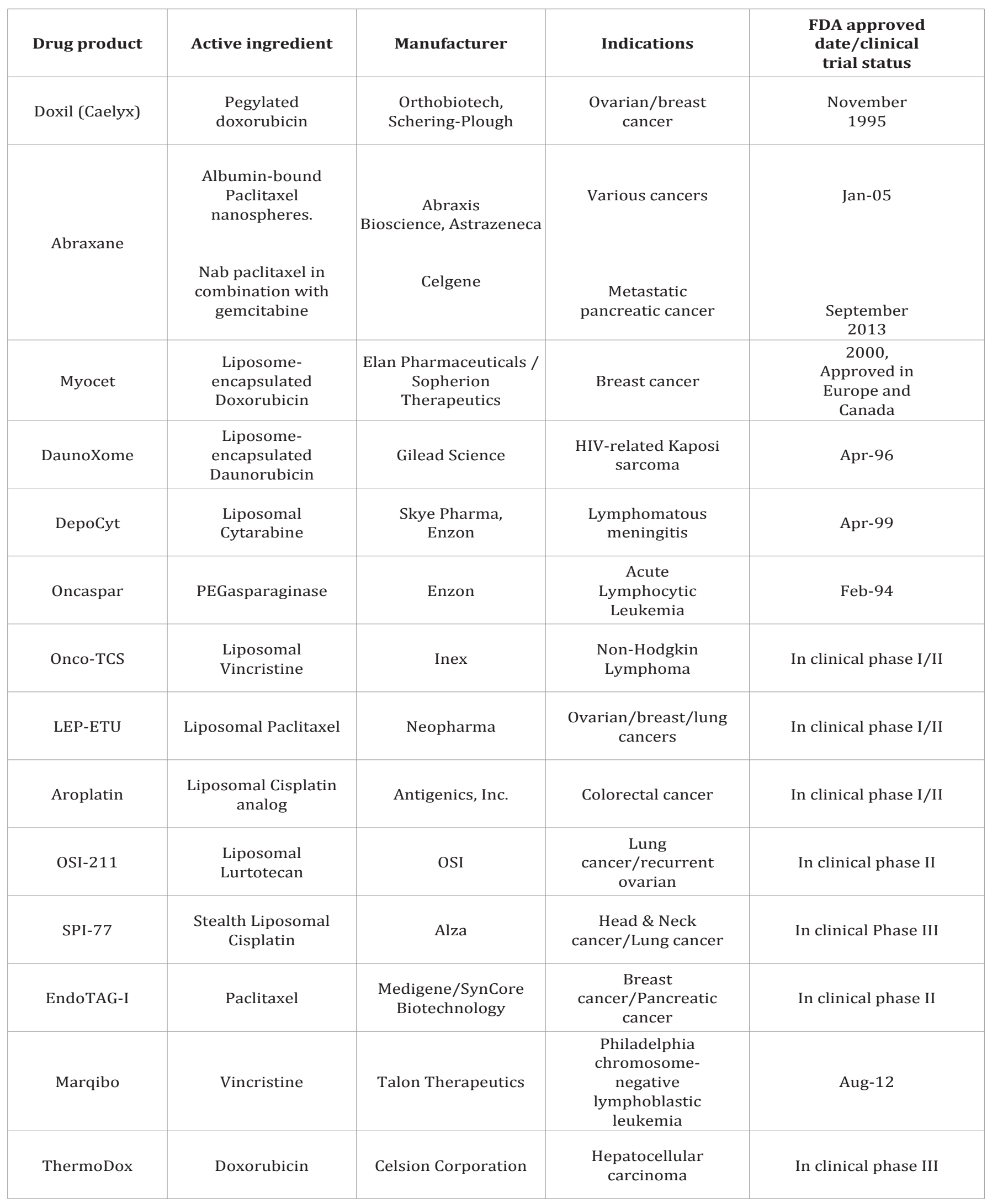




\begin{tabular}{|c|c|c|c|c|}
\hline Atragen & $\begin{array}{l}\text { Liposomal all trans- } \\
\text { retinoic acid }\end{array}$ & $\begin{array}{c}\text { Aronex } \\
\text { Pharmaceuticals }\end{array}$ & $\begin{array}{l}\text { Acute } \\
\text { promyelocytic } \\
\text { leukemia }\end{array}$ & In clinical phase II \\
\hline Lipoplatin & Liposomal Cisplatin & Regulon & $\begin{array}{c}\text { Pacreatic/ Head } \\
\text { and Neck/breast } \\
\text { cancer }\end{array}$ & In clinical phase III \\
\hline $\begin{array}{l}\text { Aurimmune } \\
\text { (CYT-6091) }\end{array}$ & $\begin{array}{l}\text { TNF- } \alpha \text { bound to } \\
\text { colloidal Gold } \\
\text { nanoparticles }\end{array}$ & $\begin{array}{l}\text { Cytimmune } \\
\text { Sciences }\end{array}$ & $\begin{array}{l}\text { Head and Neck } \\
\text { cancer }\end{array}$ & In clinical phase II \\
\hline Auroshell & Gold nanoshells & $\begin{array}{l}\text { Nanospectra } \\
\text { Biosciences }\end{array}$ & $\begin{array}{c}\text { Aurolace therapy } \\
\text { of cancer }\end{array}$ & In clinical Phase 1 \\
\hline Genexol-PM & $\begin{array}{l}\text { Paclitaxel-loaded } \\
\text { polymeric micelle }\end{array}$ & Samyang & $\begin{array}{l}\text { Breast cancer/small } \\
\text { cell lung cancer }\end{array}$ & $\begin{array}{l}\text { Marketed in } \\
\text { Europe, Korea }\end{array}$ \\
\hline Paclical & Paclitaxel micelles & $\begin{array}{c}\text { Oasmia } \\
\text { Pharmaceutical AB }\end{array}$ & Ovarian cancer & Phase III \\
\hline Narekt -102 & $\begin{array}{l}\text { Irinotecan, PEGylated } \\
\text { liposome }\end{array}$ & Nektar Therapeutics & $\begin{array}{c}\text { Breast / colorectal } \\
\text { cancer }\end{array}$ & $\begin{array}{l}\text { In clinical } \\
\text { Phase } 3\end{array}$ \\
\hline NKTR-105 & PEG-Docetaxel & Nektar Therapeutics & Solid tumors & Phase 1 \\
\hline Ontak & Diphtheria toxin and & Seragen, Inc & Cutaneous T-cell & 1999 \\
\hline
\end{tabular}

Doxorubicin hydrochloride liposome injection is currently on the FDA's drug shortage list. The U.S. Food and Drug Administration approved the first generic version of Doxil (doxorubicin hydrochloride liposome injection, February 2013) made by Sun Pharma Global FZE, a subsidiary of India's Sun Pharmaceutical Industries Ltd, to ease drug shortage. ${ }^{[28]}$ Lipodox is the second generation of pegylated liposomal doxorubicin composed of distearoyl phosphatidyl choline (DSPC) and cholesterol with surface coating of PEG. ${ }^{[38]}$ Lipodox has a circulation half-life of 65 hours. However, due to the long circulation time of the pegylated drug, stomatitis (inflammation of mucus lining) became the new dose-limiting toxicity. Doxil and lipodox (both pegylated) accumulate at tumor site by passive targeting mechanism. Unfortunately, both have more side effects than Myocet (non-pegylated doxorubicin). Moreover, liposomal formulations are more expensive than the non-liposomal drugs. The average per dose of doxil is approximately 10-20 fold higher compared to doxorubicin but a corresponding increase in patient survival has not been demonstrated. Another concern is the toxicity of liposomal formulations especially that of the pegylated liposomes, such as various skin reactions and hypersensitivity reactions.

\section{Myocet}

Myocet is a non-pegylated liposomal doxorubicin citrate made by Enzon Pharmaceuticals for Cephalon in Europe and for Sopherion Therapeutics in the United States and Canada. Myocet received Canadian regulatory approval on December 21, 2001 and is indicated for the first-line treatment of metastatic breast cancer in combination with cyclophosphamide. Myocet is also approved in Europe but not yet approved for use in the United States. It is currently being studied in combination with Herceptin (trastuzumab) and Taxol (paclitaxel) for treatment of HER2-positive metastatic breast cancer. Myocet has a different pharmacokinetic profile from doxorubicin, resulting in an improved therapeutic index (less cardiotoxicity and equal anticancer activity). ${ }^{[39]}$

The Myocet liposome is made up of egg phosphatidylcholine and cholesterol in a molar ratio of 55:45 and contains an equivalent of $50 \mathrm{mg}$ doxorubicin hydrochloride. A pH gradient method is used to encapsulate the drug which consists of maintaining an acid $\mathrm{pH}$ of 4 with citric acid in the inner compartment and a $\mathrm{pH}$ of 7 in the outer compartment where doxorubicin hydrochloride is added. The neutral form crosses the lipid layer from the outside and the protonated form remains inside the liposome. The diameter of the liposome is about $180 \mathrm{~nm}$ and the drug to lipid ratio is 0.27 . The product is supplied to the end-user as a three vial system. One of the vial contains a lyophilized red powder of doxorubicin hcl and another contains a suspension of the liposomes in citric acid and sodium chloride and the third vial contains a sodium carbonate buffer. The actual drug-loading occurs in the pharmacy by a specified procedure. The initial recommended dose of myocet is $60-75 \mathrm{mg} / \mathrm{m}^{2}$ and cyclophosphamide $(600 \mathrm{mg} /$ $\mathrm{m}^{2}$ ) every three weeks. The clearance of doxorubicin in breast cancer patients receiving Myocet was found to be 5-9 fold lower and the volume of distribution about 10-25 fold lower than in patients receiving conventional doxorubicin. The half-life has been reported to be between 16-50 hours, which is significantly longer than conventional doxorubicin. These findings are in agreement with the theoretical advantage of using a liposomal encapsulation for drug delivery. ${ }^{[40-41]}$ 


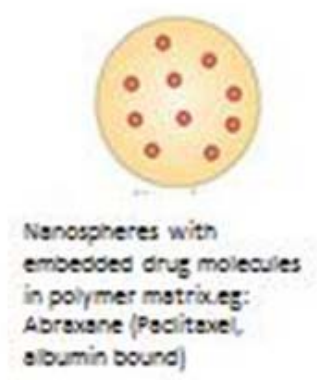

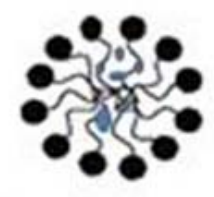

Micelles with en entropped

orugeg: Genevol-pN

(Poditoxel in oo-poirme:

micelles]

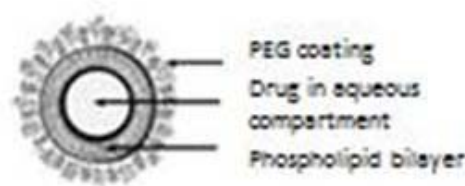

PEG costing to aroid MPS uptake.

Eg: Doxil (ptorlated fiposoma?

Doxorubicin]

Figure 1: Nanocarriers of anticancer drugs and examples of FDA approved nanomedicines.

Although Myocet and Doxil have the same API in liposomes, they are different in terms of the lipid composition, size, loading method, different plasma and tissue distribution profile as well as different dosing and safety profiles. They are certainly not bioequivalent.

\section{ThermoDox}

A strategy leading to innovative nanoformulations focuses on nanosystems that can be triggered to release its contents on exposure to external stimuli such as heat, light, ultrasound, and magnetic fields, in order to maximize drug release at the pathological site. Thermosensitive liposomes, hydrogels, dendrimers and micelles have been studied for release of drugs at sites of elevated temperature. Generally, thermosensitive liposomes make use of lipids with transition temperature between $40-45^{\circ} \mathrm{C}$. ThermoDox, a temperature-sensitive doxorubicin-pegylated liposome (Celsion, in partnership with Duke University) being developed for liver cancer, is an interesting example of stimuli-responsive nanomedicine. This new generation liposomes are being developed to release their encapsulated drug where local tissue temperatures are elevated to $39-42^{\circ} \mathrm{C}$ by application of radiofrequency, a technique referred to as radiofrequency ablation. The lipid components in the liposome undergo a gel to liquid transition at elevated temperatures, rendering it more permeable, thus releasing the drug. In addition, application of local hyperthermia results in the blood vessels within tumors to leak, thus increasing accumulation of liposomes in the tumor. ThermoDox is composed of a mixture of dipalmitoyl phosphatidylcholine (DPPC), monostearoyl phosphatidyl choline (MSPC) and PEG2000 DSPE (distearoyl phosphatidyl ethanolamine) in 90:10:4 molar ratios. DPPC has a transition temperature of $41.5^{\circ} \mathrm{C}$, therefore, it is appropriate for temperature triggered technology. For thermodox, this technology allows concentration of the drug up to 25 times more in the treatment area than IV doxorubicin, and several fold the concentration of other liposomally encapsulated doxorubicins. ${ }^{[42,43]}$ Celsion also is developing a thermosensitive liposomal Docetaxel which was evaluated in mice xenograft with lung cancer and was found to be superior in reducing the tumor volume compared to docetaxel and non-thermosensitive liposomal formulation.

\section{DaunoXome (Liposomal Daunorubicin)}

Daunoxome (Gilead sciences, now sold to Galen Pharmaceuticals)
Daunorubicin is an anthracycline antibiotic with antineoplastic activity. The anthracycline moiety is linked to an aminosugar, daunosamine via a glycosidic linkage. Daunoxome contains an aqueous solution of daunorubicin citrate encapsulated within liposomes made up of distearoylphosphatidyl choline, cholesterol and daunorubicin in a molar ratio of 10:5:1. The mean diameter is $45 \mathrm{~nm}$ and the total lipid to daunorubicin ratio is 18.7:1. The liposome injection has pH between 4.9 to 6 and the dispersion is red and translucent. It was approved by FDA in 1996 for HIV- associated Kaposi sarcoma. The liposomes are not rapidly cleared from the plasma by the Mononuclear Phagocyte System (MPS) and release of daunorubicin continues in a sustained manner. Preclinical studies indicate increased tissue concentrations of daunorubicin in tumor, brain, liver, spleen and intestine following daunoxome compared with free daunorubicin administration, but a reduced tissue concentration in cardiac tissue. ${ }^{[4]}$ In addition, studies using radio-labelled vesicles suggest selective uptake into tumor. ${ }^{[45]}$ The antitumor effects of Daunorubicin are due to intercalation into DNA and inhibition of topoisomerase II activity, resulting in decreased synthesis of both DNA and RNA. In addition, the free radical pathway that generates hydroxyl- and superoxide radicals and which peroxidizes lipids and damages cellular membranes also contributes to its antitumor activity. Pharmacokinetic studies following administration of $40 \mathrm{mg} / \mathrm{m}^{2}$ in pediatric patients revealed a mono-exponential decline in all patients and the PK parameters were not dose-dependent. The total plasma clearance was 0.423 $\mathrm{L} / \mathrm{h} / \mathrm{m}^{2}$, elimination half-life of $5.5 \mathrm{~h}$ and a volume of distribution of $3.7 \mathrm{~L} / \mathrm{m}^{2} .^{[46,47]}$ These differences in the volume of distribution and clearance result in higher daunorubicin exposure than with daunorubicin hcl.<smiles>COc1cccc2c1C(=O)c1c(O)c3c(c(O)c1C2=O)C[C@@](O)(C(C)=O)C[C@@H]3O[C@H]1C[C@@H](N)[C@H](O)[C@H](C)O1</smiles>

\section{Daunorubicin}

\section{Abraxane (Albumin-bound Paclitaxel, Paclitaxel protein bound)}

Paclitaxel (Taxol, Bristol-Myers Squibb) belongs to an 
important class of antitumor agents called taxanes.. Paclitaxel is a semisynthetic diterpenoid taxane derivative used for the treatment of a variety of malignancies including breast, lung, non-small cell lung cancer and ovarian cancer. Taxanes are cell cycle-specific agents and bind with high affinity to microtubules resulting in inhibition of mitosis and cell death.

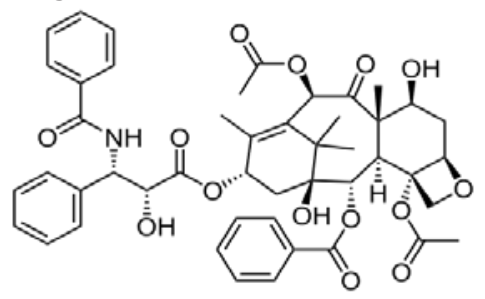

\section{Paclitaxel}

Taxanes are highly hydrophobic molecules and therefore have very low solubility in water. In order to solubilize paclitaxel, a derivative of castor oil, called polyethoxylated castor oil (cremophore EL, a non-ionic surfactant) and ethanol (50:50) is used. However, the use of the solventbased formulation is associated with serious and dose-limiting toxicities. Cremophore itself causes hypersensitivity reactions (premedication with a steroid (oral dexamethasone) and antihistamine (diphenhydramine) was required) and is known to leach out plasticizers from standard intravenous injection tubing, necessitating special infusion set. ${ }^{\left[{ }^{[8]}\right]}$ The albumin bound paclitaxel, Abraxane, however, do not contain cremophore (Figure 1). Therefore, no premedication or special tubing is required to administer abraxane. Abraxane (Abraxis Bioscience/ Celgene) was approved by the FDA in January 2005 for treatment of metastatic breast cancer and in October 2012 as a first line treatment for advanced non-small cell lung cancer in combination with carboplatin for patients who are not candidates for curative surgery or radiation therapy. Abraxane has also shown promise for treatment of advanced pancreatic cancer ${ }^{[49]}$ and the US FDA has expanded approval to include advanced pancreatic cancer in 2013. Abraxane formulation uses nanotechnology to combine human albumin with paclitaxel allowing for the delivery of an insoluble drug in the form of nanospheres (130 nanometers in diameter). Albumin is an ideal carrier for drug delivery because of its preferential uptake in tumor and inflamed tissue, ready availability, biodegradability, and lack of toxicity and immunogenicity. Albumin-bound paclitaxel is the first biologic chemotherapeutic compound to exploit the gp60 receptor (albondin)-mediated pathway in endothelial cell walls of tumor microvessels to achieve enhanced intra-tumoral concentrations. Gp60 (albondin) is a 60-kda glycoprotein localised on the endothelial cell surface that binds to native albumin with a high affinity. The accumulation of albumin and albumin-bound drugs in the tumor interstitium is further facilitated by SPARC (Secreted Protein, Acidic and Rich in Cysteine) over- expressed in multiple type of tumors including breast, prostate, gastric, lung and kidney. More importantly, it is shown that increased SPARC levels in tumors' correlate with enhanced response to Abraxane.

Abraxane formulation has increased the bioavailability of paclitaxel and resulted in higher intra-tumor concentrations facilitated by albumin-receptor (gp60) mediated endothelial transcytosis. ${ }^{[50-52]}$ Nanoparticle albumin bound (NAB) paclitaxel is administered as a suspension intravenously $\left(260 \mathrm{mg} / \mathrm{m}^{2}\right.$ as infusion for 30 minutes). $N a b$ paclitaxel can be reconstituted in normal saline at concentrations of $2-10 \mathrm{mg} / \mathrm{ml}$, compared with $0.3-1.2 \mathrm{mg} / \mathrm{ml}$ for paclitaxel. It undergoes biphasic elimination (two-compartment model of disposition) with a terminal halflife of 27 hours (5.8 hours for paclitaxel). The clearance is $43 \%$ slower $\left(15 \mathrm{~L} / \mathrm{h} / \mathrm{m}^{2}\right)$ and the mean volume of distribution is 632 $\mathrm{L} / \mathrm{m}^{2}$ (indicating extensive extravascular distribution). The drug exposure (AUC) was proportional to the dose in the range of 80$375 \mathrm{mg} / \mathrm{m}^{2}$

Another nanocarrier for paclitaxel is being developed by Cell therapeutics, called "Paclitaxel poliglumex (PPX)". This product is also known as Xyotax and Opaxio or CT 2103. It is a Soluble, large macromolecular conjugate of paclitaxel and poly-L-Glutamic acid and contains $37 \%$ of Paclitaxel. The polymer, poly-L glutamic acid, is biodegradable. When bound to the polymer, paclitaxel is inactive, potentially sparing healthy tissues from exposure to high level of paclitaxel and its associated toxicities. PPX, because of its large size, accumulates in tumor tissues by taking advantage of the enhanced permeability of tumor vasculature and lack of lymphatic drainage. The drug is released from the polymeric backbone by the intracellular lysosomal enzyme protease, cathepsin B, which is up-regulated in many tumor types. ${ }^{[53,54]}$ Preclinical studies in animal tumor models demonstrate that PPX is more effective than standard paclitaxel and is associated with prolonged tumor exposure to active drug while minimizing systemic exposure. Phase 1 and 2 clinical studies with PPX showed encouraging outcomes compared to standard taxanes with reduced neutropenia and alopecia and allowed a more convenient administration schedule without the need for routine premedications. $^{[53]}$

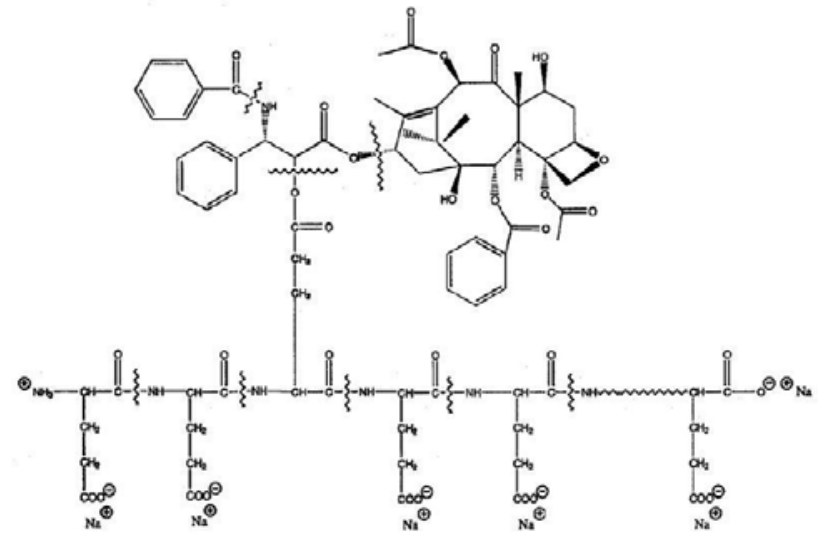

\section{Polyglumex}

Human pharmacokinetic studies are consistent with prolonged tumor exposure to active drug and limited systemic exposure. Based on these results, three ongoing randomized phase III trials were initiated to test the efficacy of paclitaxel poliglumex in patients with advanced non-small cell lung carcinoma. ${ }^{[55-58]}$ 
Another agent under investigation and now in phase IV clinical trial is Genexol-PM (Samyang Biopharmaceuticals, South Korea) which consists of 20-50 nm micelles formed by the selfassembly of polyethylene glycol and polylactide polymers (Figure 1). The core of these micelles contains paclitaxel. ${ }^{[59]}$ The copolymer increases the water-solubility of paclitaxel and allows delivery of higher doses than those achievable with paclitaxel alone. Genexol-PM was launched in Korean market for breast cancer and small cell lung cancer in February 2007.

\section{EndoTAG-1}

Medigene AG (a biotechnology company in Munich,Germany) obtained U.S. patent for EndoTag®-1 (formerly named lipopack) in May 2012 for the Treatment of Triple-Negative Breast cancer. Triple negative breast tumors do not have any HER2 receptors or hormone receptors for estrogen or progesterone. There are very few treatment options available for this type of cancer since the conventional antihormonal therapy or treatments targeting HER2 are not appropriate. Endotag ${ }^{\circledR}-1$ is a cationic liposome containing paclitaxel. The liposome is formed from 1,2 dioleoyl-3-trimethylammonium-propane (DOTAP) and 1,2 dioleoyl-sn-glycero-3-phosphocholine (DOPC) and paclitaxel in a molar ratio of 50:45:5. The diameter is about $220 \mathrm{~nm}$. Due to the positively charged lipids, endotag ${ }^{\circledR}-1$ interacts with newly developing, negatively charged endothelial cells required for the growth of tumor blood vessels. The endotag ${ }^{\circledR}-1$ targets the tumor vasculature without affecting the supply to endothelial cells of healthy tissue. By doing this, it prevent the formation of new tumor blood vessels, incr?ases tumor vascular permeability and inhibit tumor growth. ${ }^{[60]}$ A controlled phase II clinical trial for pancreatic cancer showed significantly increased survival rates in patients treated with endotag- 1 and gemcitabine combination therapy. A phase II trial in triple negative breast cancer also showed a positive efficacy trend of the combination therapy. European and US authorities have granted orphan drug status for endotag-1 in the treatment of pancreatic cancer.

\section{Lipoplatin (Liposomal cisplatin)}

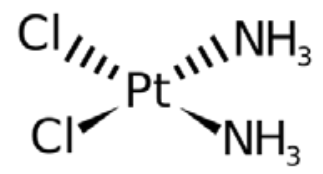

Lipoplatin is a liposomally-encapsulated (average diameter, $110 \mathrm{~nm}$ ) drug product of the FDA-approved cytotoxic agent cisplatin. The major concern about the use of cisplatin and other platinum compounds is nephrotoxicity. Patients receiving these agents need to be hydrated to prevent renal damage. In the Lipoplatin product, cisplatin (cis-diamino dichloro-platinum) is encapsulated in a liposome shell composed of dipalmitoyl phosphatidyl glycerol, soy phosphatidyl choline, cholesterol and methoxy-polyethylene glycol-distearoyl phosphatidylethanolamine lipid conjugate. The ratio of cisplatin to lipids is 8.9\%:91.1\% (w/w) ${ }^{[61,62]}$ Lipoplatin is used against pancreatic cancer in combination with gemcitabine as first line treatment. It has shown superiority to cisplatin in combination with paclitaxel as a chemotherapy regimen in non-small cell lung cancer (NSCLC) adenocarcinomas. Lipoplatin accumulates in the cancer tissues by the extravasation of the nanoparticles through the defective vasculature of the tumor tissue during neoangiogenesis. Lipoplatin had mild hematological and gastrointestinal toxicity and did not show nephron, neuro, oto- toxicity or any other side effects characteristic of cisplatin. The half-life of total platinum in the human plasma was 60-117 hours compared to 6 hours for cisplatin. ${ }^{[63]}$ Human studies have shown 40- to 200 -fold higher platinum concentration compared to the adjacent normal tissue in specimens from human biopsies $20 \mathrm{~h}$ post-infusion of the drug. The clinical development of Lipoplatin in adenocarcinomas establishes this drug as the most active platinum drug with significantly lower side effects. Lipoplatin, under the name Nanoplatin, received in 2009 the consent of European medicine agency to be tested as first line against non-squamous NSCLC mainly composed of adenocarcinomas.

\section{OncoTCS (Vincristine)}

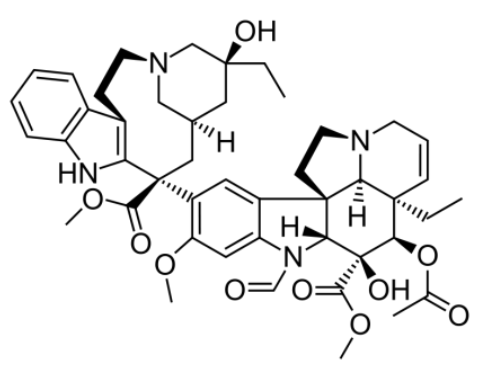

Vincristine (VCR) and Vinblastine (VLB) are alkaloids obtained from the flowering plant. Catharanthus roseus (Periwinkle, also called the Madagascar periwinkle). Both have powerful anticancer activity. On August 9, 2012, the Food and Drug Administration (FDA) granted accelerated approval for vincristine sulfate liposome injection (Marqibo ${ }^{\circledR}$, made by Talon Therapeutics, Inc.) For the treatment of adult patients with Philadelphia chromosome-negative ( $\mathrm{Ph}-$ ) acute lymphoblastic leukemia (ALL). Vincristine administered as the liposomal formulation exhibits a lower clearance and higher AUC compared with conventional VCR. ${ }^{[64,65]}$ INEX Pharmaceuticals is developing a liposomal formulation of vincristine (Onco TCS, vincacine, VSLI, Vincristine sulfate liposomes for injection) for the treatment of relapsed aggressive non-Hodgkin's lymphoma (NHL) and other cancers. (Inex is a Canadian biopharmaceutical company developing and commercializing proprietory drugs and drug delivery systems to improve treatment of cancer). Vincristine is being developed using INEX's proprietary drugdelivery technology platform called the "transmembrane carrier systems" (TCS). Liposomal vincristine is expected to have certain advantages over the existing standard preparation because vincristine in liposomes enables the drug to increase blood circulation time, increase the drug accumulation in the blood, increase drug accumulation in the tumor, and be released over an extended period. 


\section{Vinorelbine}

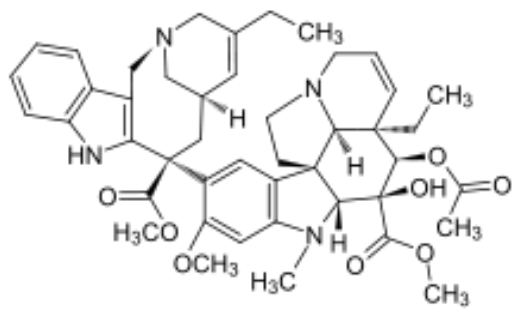

Vinorelbine, a microtubule inhibitor, is a semisynthetic vinca alkaloid shown to be useful for treatment of a variety of malignancies, such as small cell lung, breast, ovarian, head and neck, cervical and kaposi sarcoma. A new formulation of vinorelbine, called Allocrest, consists of vinorelbine tartrate encapsulated in the aqueous core of a liposome (sphingomyelin-based liposomes called Optisome ${ }^{\mathrm{TM}}$ ). This formulation has been developed to achieve targeted delivery of the drug in high concentration in the tumor and also sustained release. In animal models, the Optisome technology resulted in prolonged plasma circulation (100-fold increased area under the concentration- time curve) and 9.5fold enhanced cancer tissue drug penetration and accumulation as compared to that achievable with un-encapsulated, standard vinorelbine. The high intra-tumoral vinorelbine concentration is expected to improve cancer cell killing beyond the capability of standard vinorelbine. The prospect of providing enhanced anti-cancer activity without enhanced toxicity is of particular importance in the treatment of elderly cancer patients and those with marginal performance status. Nano vinorelbine (nanovnb) was found to be active against human breast cancer and lung cancer xenograft. ${ }^{[66]}$

\section{DepoCyt (Liposomal Cytarabine)}

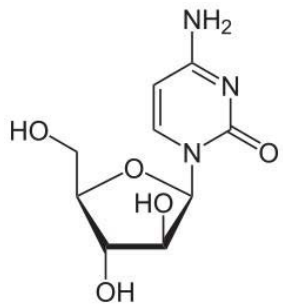

Cytarabine

DepoCyt is a sustained -release liposomal formulation of cytarabine, prepared by a unique proprietary technology called depofoam. Unlike the usual unilamellar or multilamellar liposomes, the depofoam is a multivesicular system containing hundreds of water-filled compartments separated by a lipid bilayer. This structure allows encapsulation of large quantities of drugs and ensures prolonged release. ${ }^{[67-69]}$

Cytarabine liposome injection (DepoCyt, Enzon Pharmaceuticals), was given full approval by the FDA in April 2007 for the treatment of lymphomatous meningitis, a life-threatening complication of lymphoma. It was originally approved in April 1999 under the accelerated approval regulations based on increased response rate compared to the unencapsulated drug. It is the only liposomal drug for intrathecal use and should be administered only under supervision of a qualified physician. Systemic exposure to cytarabine is negligible when the liposome is given intrathecally. Cytarabine is a cell-cycle specific antineoplastic agent affecting cells only in the s-phase of cell divison. Depofoam-encapsulation has been shown to result in a sustained-release lasting several days to weeks (Depocyt has a half-life of up to 82.4 hours compared to 3.4 hours for the unencapsulated drug) after nonvascular administration. Depocyt is well distributed throughout the cerebrospinal fluid to provide continuous tumor cells with complete exposure to cytarabine. (The routes of administration most viable for delivery of drugs via depofoam formulations include intrathecal, epidural, subcutaneous, intramuscular, intraarticular, and intraocular). Depofoam particles are distinguished structurally from unilamellar vesicles, multilamellar vesicles, and niosomes in that each particle comprises a set of closely packed non-concentric vesicles. The particles are tens of microns in diameter and have large trapped volume, thereby affording delivery of large quantities of drugs in the encapsulated form in a small volume of injection. A number of methods based on a manipulation of the lipid and aqueous composition can be used to control the rate of sustained-release from a few days to several weeks.

The liposome contains dioleoyl phosphatidyl choline, dipalmitoyl phosphatidyl glycerol, cholesterol and triolein. It is a long-acting, preservative-free formulation. The pharmacokinetic advantage of this formulation was that the terminal halflife was 40 times longer than that of standard cytarabine. [70] Chemical arachnoiditis, manifested primarily by nausea, vomiting, headache and fever, has been a common adverse event following administration of DepoCyt. The incidence and severity of arachnoiditis can be reduced by the co-administration of dexamethasone. Therefore, it is recommended that all patients receiving DepoCyt should be treated concurrently with dexamethasone. Cytarabine can cause fetal harm if a pregnant woman is exposed to the drug systemically. However, the concern for fetal harm following intrathecal DepoCyt administration is low because systemic exposure to cytarabine is negligible. ${ }^{[71]}$

\section{Annamycin}<smiles>COc1cccc2c1C(=O)c1c(O)c3c(c(O)c1C2=O)C[C@@](O)(C(=O)CO)C[C@]3(O)O[C@@H]1O[C@H](C)[C@@H](O)[C@H](O)[C@H]1I</smiles><smiles>COc1cccc2c1C(=O)c1c(O)c3c(c(O)c1C2=O)C[C@@](O)(C(=O)CO)C[C@@H]3O[C@H]1C[C@H](N)[C@@H](O)C(C)O1</smiles> 
Annamycin is a semisynthetic doxorubicin analog developed at the M.D Anderson Cancer Institute in Houston, Texas. Annamycin was initially investigated as an antineoplastic without the multidrug resistance related to p-glycoprotein. The p-glycoprotein is a major factor limiting the efficacy of anthracycline group of drugs. ${ }^{[72]}$ As shown in the structure above, annamycin does not have the amino group in the sugar moiety as in doxorubicin. Removal of the amino group seems to reduce the cardiac toxicity without changing its antitumor properties. Annamycin intercalates into DNA and inhibits topoisomerase II. This results in the inhibition of DNA replication and repair as well as RNA and protein synthesis. Liposomal annamycin is less toxic and shows improved antitumor activity compared to annamycin and is indicated for breast cancer. ${ }^{[73,74]}$

\section{Oncaspar (Asparaginase)}<smiles>NC(=O)C[C@H](N)C(=O)O</smiles>

\section{Asparagine}

Leukemic cells are unable to synthesize asparagine due to lack of asparagine synthetase and are dependent on exogenous source of asparagine for survival. Asparaginase is the enzyme that depletes the amino acid asparagine. Depletion (starving the leukemic cells) of asparagine ultimately results in leukemic cell death. Normal cells are less affected because of their ability to synthesize asparagine. L-asparaginase has been an important component in the treatment of acute lymphoblastic leukemia (ALL) ${ }^{[75,76]}$ Oncaspar is a modified form of the enzyme, L-asparginase. The tetrameric enzyme, derived from E.coli, is covalently conjugated with monomethoxy polyethylene glycol (mpeg). Approximately 69-82 molecules of mpeg are linked to L-Asparaginase. The molecular weight of each mpeg molecule is 5 kda. PEG asparaginase (Enzon) was approved by FDA in July, 1994 for use in acute lymphoblastic leukemia (ALL). Patients with allergy to the drug were unable to receive L-Asparaginase. The use of Oncaspar in place of L-asparaginase, markedly reduced the number of drug injections required from 21 injections of Elspar (L-asparaginase), to three injections with Oncaspar over the 20-week course of treatment. Through the process of pegylation, the half-life of L-asparaginase is significantly increased (approximately 6 days) and the L-asparaginase activity is sustained. ${ }^{[77]}$ Oncaspar provides patients the full benefits of asparaginase therapy with an enhanced convenience over native L-asparaginase (non-pegylated form). Oncaspar can be administered through intramuscular (IM) injection or intravenous (IV) infusion. When utilized as a component of induction therapy for ALL, a single dose of Oncaspar achieved similar levels of asparagine depletion as 9 doses of native L-asparaginase. ${ }^{[77]}$

\section{Camptothecins}

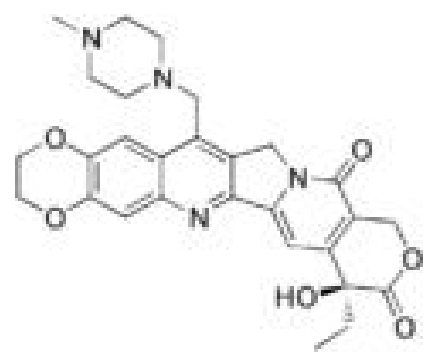

Lurtotecan<smiles>CC[C@@]1(O)C(=O)OCc2c1cc1n(c2=O)Cc2cc3ccccc3nc2-1</smiles>

Lurtotecan is a potent semisynthetic derivative of camptothecin. This analog is water-soluble. Lurtotecan is a topoisomerase inhibitor used for epithelial ovarian cancer. Lurtotecan has been formulated into unilamellar liposomes. Pharmacokinetic studies in nude mice have demonstrated increased plasma residence time and 1500-fold increase in AUC. ${ }^{78,79]}$

Irinotecan, another agent of this group added to fluorouracil (FU), resulted in the first major advance in many years in the treatment of colorectal cancer and the combined administration of these drugs became the standard of care. ${ }^{[00]}$ Irinotecan (Camptostar,NKTR-102) is being developed by Nektar Therapeutics as a pegylated liposomal formulation for the treatment of colorectal cancer<smiles>CCc1c2c(nc3ccc(OC(=O)N4CCC(N5CCCCC5)CC4)cc13)-c1cc3c(c(=O)n1C2)COC(=O)[C@@]3(O)CC</smiles>

Irinotecan (NKTR-102)

NKTR-102 is a new therapeutic option in development for advanced breast cancer. It is the first long-acting topoisomerase I inhibitor. NKTR-102 is believed to penetrate the vasculature of the tumor environment more readily than normal vasculature, increasing the concentration of active drug within tumor tissue to enhance anti-tumor activity. The unique PK profile of NKTR102 provides continuous exposure of active drug throughout the entire chemotherapy cycle, with reduced peak exposures that can be associated with toxicities. NKTR-102 is also being evaluated 
for the treatment of ovarian, glioma and lung cancers. The most significant adverse effects of irinotecan are severe diarrhea and extreme suppression of the immune system. Irinotecan received accelerated approval by the (FDA) in 1996 and full approval in 1998. During development, it was known as CPT-11.<smiles>CC1=C(/C=C/C(C)=C/C=C/C(C)=C/C(=O)O)C(C)(C)CCC1</smiles>

\section{All-trans retinoic acid}

ATRA or ATRAGEN (Liposomal tretinoin, lipoatra, AR-623) is a vitamin A acid or all trans-retinoic acid. Retinoids bind to and activate retinoic acid receptors resulting in gene expressions leading to cell differentiation, decreased cell proliferation and inhibition of carcinogenesis. ATRA also inhibits telomerase, telomere shortening and eventual apoptosis of some tumor cell types. ATRA is used to treat acute promyelocytic leukemia (APL, APML) in patients who have not responded to other treatments. One of the disadvantages of oral ATRA is its very poor bioavailability. It is almost insoluble in aqueous medium with highly variable absorption from the intestine. The liposomal delivery system of ATRA alters the drug's pharmacokinetics and decreases toxicity associated with oral administration. Following (iv) injection, this formulation is able to bypass the hepatic clearance mechanism that metabolizes the oral formulation of ATRA. Additionally, in vitro studies have shown that liposomal ATRA has a greater anti-proliferative effect on neoplastic cells as compared to free ATRA. It can inhibit the proliferation of lymphoma cells in a dose-dependent manner by inducing apoptosis. ${ }^{[81-83]}$ It has also been shown that the iv administration of liposomal retinoic acid in human subjects resulted in a 13-15 fold higher plasma concentration than retinoic acid. ${ }^{[83]}$

\section{Gold Nanoparticles}

One of the most studied nanotechnology-based nanoparticles is the gold nanoparticles (gnps). Gnps are potential drug carriers, photothermal agents, radiosensitizers, contrast agents and have also have shown promise for cancer therapy. There are four types of gold nanoparticles: gold colloid, gold-silica nanoshells, gold-gold sulfide nanoparticles, and gold nanorods. Each of these four particles has demonstrated success in both optical imaging and photothermal ablation of cancer. There are several methods for the preparation of gold nanoparticles. One of the methods involves reduction of chloroauric acid with sodium citrate. Varying the molar ratio of citrate to chloroauric acid results in variation of gnps sizes, from 50-150 $\mathrm{nm}$. The nanoparticles are stabilized by coating with polyethylene glycol and anticancer drugs can be attached to the coated nanoparticles. Gnps exhibit unique physicochemical properties: their optical properties and ability to bind to amino and thiol groups permits surface functionalization for various biomedical applications. Tumorspecific ligands such as transferrin, folic acid, monoclonal antibodies, and tumor necrosis factor have been attached to surface of gold nanoparticles combined with a chemotherapeutic agent for specific delivery to tumor. Aurimmune (Cytimmune Sciences, Rockville, MD) is a $27 \mathrm{~nm}$ gold nanoparticle coated with thiolated PEG and attached to recombinant human tumor necrosis factor $\alpha$ (TNF- $\alpha$ ) which has the ability to accumulate in the tumor and exert its antitumor activity. TNF- $\alpha$ is a multifunctional cytokine known to have both cytotoxic and immunomodulatory effects. Prior to aurimmune, attempts to use TNF- $\alpha$ in adequate doses for its anticancer response were not successful due to doselimiting toxicity, such as hypotension and nausea. However, no dose-limiting toxicity was observed when dose levels as high as 500-600 microgram $/ \mathrm{m}^{2}$ of TNF- $\alpha$ was administered to patients with solid tumors. The main side effect was only a grade 2 fever. Intracellular gnps were detectable in post-treatment tumor biopsies but not in healthy tissues. A recent study reported the amplification of biochemical action of Au-TNF by laser-induced photothermal effect in target tumor. ${ }^{[84]}$ Another device that has advanced to clinical trials is a nanoparticle of silica coated with a thin layer of gold, called AuroShell (Nanospectra Bioscience Inc, Houston, Texas). Photothermal therapy of head and neck cancer is possible when the AuroShell is irradiated with near infra-red rays from a laser. Gold nanoshells are more efficient in converting the incident light in to heat than nanoparticles. This is referred to as AuroLase therapy. ${ }^{[84-89]}$ The potential benefit of AuroLase therapy is the highly selective and rapid tumor destruction with minimal damage to surrounding healthy tissue. Preclinical studies have demonstrated that AuroLase Therapy is effective and causes no detectable systemic toxicity. Additionally, this therapeutic device may be used in combination with standard chemotherapy and radiation.

\section{SiRNA Delivery}

RNA interference, or gene silencing, is one of the most important discoveries in the field of biology in the last decade for which a Nobel Prize in medicine was awarded in 2006. RNA interference is a natural phenomenon which can be used to selectively turn off the genes expressed in some diseases. Molecular therapy using small interfering RNA (siRNA, synthetic RNA molecules designed to suppress production of specific proteins) has shown great therapeutic potential for diseases caused by abnormal gene over-expression or mutation. When the siRNA-based therapeutics are introduced in to the cell cytoplasm, they are guided to target protein mRNA which is then cut and destroyed, preventing subsequent production of target protein. The challenges in developing effective delivery systems include the instability of RNA in the blood and its inability to enter the cell cytoplasm. Recent developments in nanotechnology is making it possible to deliver these gene silencing agents in the true nanosize of 20-40 nm that displays favorable EPR effect and free of antigenicity. ${ }^{[90-92]}$ Currently there are several phase 1 clinical trials underway using nanotechnology based delivery of siRNA including the following: CALLA 01(Calando Pharmaceuticals, a subsidiary of Arrowhead Research Corporation, a biotech company developing targeted RNAi therapeutics) using cyclodextrin nanoparticles attached to transferrin and coated with PEG for solid tumors; Atu027 (Silence Therapeutics, London, UK, now merged with Intradigm 
Corporation, USA) using cationic lipids to form lipoplexes and is aimed at Protein Kinase N3(PKN3), a key factor for cancer progression and metastasis; TKM 080301 (Tekmira Pharma) targeting polo-kinase of solid tumors using lipid nanoparticles (LNP Technology).$^{\left[{ }^{[2]}\right]}$ Various in vivo delivery strategies for siRNA including chemical modification, conjugation, lipid-based techniques, polymer-based nanosystems and physical methods, have been reviewed. ${ }^{[93]}$

\section{Conclusion}

Nanomedicines at present are in an early stage of development. They have the potential, however, to overcome the limitations of conventional cancer chemotherapy by their ability to selectively target the cancer cells without doing too much damage to healthy tissue. Properly designed nanoparticles have the ability to accumulate in tumors either by passive or active targeting and enhance the cytotoxic effects of antitumor agents. Several anticancer drugs in Nano formulations have been evaluated and a few are already approved for clinical use and others are undergoing phase $2 / 3$ clinical trials. Although the nanomedicines have numerous advantages compared to conventional chemotherapy, there are concerns about their potential for toxicity to patients and to the environment in addition to the high cost of production and premature drug release. On the other hand, nanotechnology offers the opportunity to reformulate the discontinued drugs because of poor bioavailability, lack of selectivity to desired target or extreme toxicity. It is also known that drug loaded nanoparticles evade the efflux mechanism, maintain a high concentration within tumor cells and therefore avoid development of resistance.

\section{References}

1. Moghimi SM, Hunter AC, Murray JC. Nanomedicine: current status and future prospects. FASEB J2005;19(3):311-330.

2. Wilkinson JM. Nanotechnology applications in medicine. Med Device Technol2003;14(5):29-31.

3. Dan Peer, Karp JM, Hong S, Farokhzad OC, Margalit R, Langer R. Nanocarriers as an emerging platform for cancer therapy. Nature nanotechnology2007;2:751-760.

4. Martin CR. Welcome to nanomedicine. Nanomedicine2006;1(1):5-5.

5. Fernandez A, Manchanda R, mcgoran A. Theranostic applications of nanomaterials in cancer therapy: Drug delivery, imageguided therapy and multifunctional platforms. Appl Biochem Biotechnol2011;165(7-8):1628-51.

6. Jain KK. Role of nanodiagnostics in personalized cancer therapy. Clin Lab Med2012;32(1):15-31.

7. Omedi Y. Smart Multifunctional Theranostics: Simultaneous Diagnosis and Therapy of Cancer.Bioimpacts2011;1(3):145-147.

8. Torchilin VP. Multifunctional nanocarriers. Adv Drug Deliv Rev2006;58:1532-1555.

9. $\mathrm{Hu}$ CM, Zhang L. Therapeutic nanoparticles to combat cancer drug resistance. Current Drug Metabolism2009;10(8):836 -841.

10.Xiaowei D, Russel JM. Nanomedicinal strategies to treat multidrug resistant tumors: current progress. Nanomedicine (Lond)2010;5(4):597-615.

11. Sadava D, Coleman A, Kane SE. Liposomal daunorubicin overcomes drug resistance in human breast, ovarian and lung carcinoma cells. $J$ Liposome Res2002;12(4):301-309.

12. Rahman A, Husain SR, Sidiqqui J et al. Liposome-mediated modulation of multidrug resistance in human HL-60 leukemia cells. J Natl Cancer Inst1992;84(24):1909-1915.

13. Meyer LD, Shabbits JA. The role of liposomal drug delivery in molecular and pharmacological strategies to overcome multidrug resistance. Cancer Metastasis Review2001;20(1-2): 87-93.

14. Bawa R. Nanoparticle-based Therapeutics in Humans: A survey. Nanotechnology law \& business2008;5(2):135-55.

15. Giovanni Tosi, Luca Constantino, Barbara Ruozi, Flavio Forni, Maria Angela Vandelli. Polymeric nanoparticles for drug delivery to central nervous system. Exp Opin Drug Deliv2008;5(2):155-174.

16. Danhier F, Feron O, Preat V. To exploit the tumor microenvironment; Passive and active targeting of nanocarriers for anticancer drug delivery. J Control Release2010;148:135-46.

17.Gref R, Domb A, Quellec P et al. The controlled intravenous delivery of drugs using PEG coated sterically stabilized nanospheres. Adv Drug Deliv Rev1995;16(2-3):215-33.

18. Illum L, David SS, Muller RH, Mak E, West P. The organ distribution and circulation time of intravenously injected colloidal carriers sterically stabilized with a block coplolymer-poloxamine. Life Sci1987;40(4):367-74.

19. Torchilin VP. Targeted pharmaceutical nanocarriers for cancer therapy and imaging. AAPS J2007;9(2):E128-E147.

20.Suzuki R,Takizawa T. Kuwata Y et al. Effective anti-tumor activity of oxaliplatin encapsulated in transferrin-PEG-liposome. Int $J$ Pharm2008;346(1-2):143-150.

21. Gaspar MM, Radomska A, Gobbo AL, Gobbo OL, Bakowsky U, Radomski MW, Ehrhardt C. Targeted delivery of transferrin conjugated liposomes to an orthotopic model of lung cancer in nude mouse. Journal of aerosol medicine and pulmonary drug delivery2012;25:1-9.

22. Vandana Soni V, Jain SK, Kohli DV. Potential of transferrin and transferrin conjugates of liposomes in drug delivery and targeting. American Journal of Drug Delivery2005;3(3):155-170.

23. Daniels TR, Bernabeu E, Rodrigueez JA et al. Transferrin receptors and the targeted delivery of therapeutic agents against cancer. Biochim Biophys Acta2012;1820(3):291-317.

24. Lu Y, Low PS. Folate- mediated delivery of macromolecular anticancer drug therapeutic agents. Adv Drug Deliv Rev2012;54(5):675-93.

25. Hilgenbrink, Low PS. Folate receptor-mediated drug targeting: from therapeutics to diagnostics. J Pharm Sci2005;94(10):2135-46.

26. Bast RC, Feeney M, Lazaus H, L M Nadler, R B Colvin, R C Knapp. Reactivity of a monoclonal antibody with human ovarian carcinoma. J Clin Invest1981;68(5):1331-7.

27. Moor RG, maclaughlan S. Current clinical use of biomarkers for epithelial ovarian cancer. Curr Opin Oncol 2010;22(5):492-7.

28. Gopalakrishna pillai, Maria LC Coronel. Scienece and technology of the emerging nanomedicines in cancer therapy: A primer for physicians and pharmacists. SAGE Open Medicine2013;1.

29. Gewirtz DA. A critical evaluation of the mechanisms of action proposed for the antitumor effects of the anthracycline antibiotics adriamycin and daunorubicin. Biochem Pharmacol1999;57(7):727-741.

30. Park JW. Liposome-based drug delivery in breast cancer treatment 


\section{Breast Cancer Res2002;4(3):95-99.}

31. Gordon AN, Granai CO, Rose PG et al. Phase II study of liposoma doxorubicin in platinum- and paclitaxel-refractory epithelial ovarian cancer. J Clin Oncol2000;18(17):3093-3100.

32. Gordon AN, Fleagle JT, Guthrie D, Parkin DE, Gore ME, Lacave AJ. Recurrent epithelial ovarian carcinoma: A randomized phase III study of pegylated liposomal doxorubicin versus topotecan. J Clin Oncol2001;19:3312-332.

33. Rahman AM, Yusuf SW, Ewer MS. Anthracycline-induced cardiotoxicity and the cardiac sparing effect of liposomal formulation. Int J Nanomedicine2007;2(4):567-583.

34. O'Brian ME, Wigler N, Inbar M et al. CAELYX Breast Cancer Study Group. Reduced cardiotoxicity and comparable efficacy in a phase III trial of pegylated liposomal doxorubicin HCL (CAELYX/DOXIL) versus conventional doxorubicin for first-line treatment of metastatic breast cancer. Ann Oncol2004;15(3):440-449.

35. Hong RL. Liposomal anticancer drug researches the myth of long circulation. J Chinese Oncol Soc2004;20(2):10-21.

36. Klaus M, Niemann B, Massing $U$ et al. Pharmacokinetics of liposomal doxorubicin (TLC-D99; Myocet in patients with solid tumors: an open-label, single-dose study. Cancer Chemother Pharmacol2004;54(6):514-24.

37. Working PK, Newman MS, Huang SK, Mayhew E, Vaage J, Lasic DD. Pharmacokinetics, Biodistribution and Therapeutic Efficacy of Doxorubicin Encapsulated in Stealth® Liposomes (Doxil@). Journal of Liposome Research1994;4(1):667-687.

38. Johnston SR, Core ME. Caelyx: phase II studies in ovarian cancer. Eur J Cancer2001; 37(suppl.9):S8-14.

39. Leonard RCF, Williams S, Tulpule A, Levine AM, Oliveros S. Improving the therapeutic index of anthracycline chemotherapy: Focus on liposomal doxorubicin (Myocet ${ }^{\mathrm{TM}}$ ). The breast2009;18(4):218-224.

40.Gabizon A, Shmeeda H, Barenholz Y. Pharmacokinetics of pegylated doxorubicin: review of animal and human studies. Cli Pharmacokinet2003;42(5):419-36.

41. Lao J, Madani J, Puértolas T et al. Liposomal Doxorubicin in the treatment of breast cancer patients: a review. J Drug Deliv2013.

42. Swenson CE, Bolcsak LE, Batist G, et al.Pharmacokinetics of doxorubicin administered i.v. As Myocet (TLC D-99; liposome-encapsulated doxorubicin citrate) compared with conventional doxorubicin when given in combination with cyclophosphamide in patients with metastatic breast cancer. Anticancer Drugs2003;14(3):239-46.

43. Slingerland M, Guchelaar HJ, Gelderblom H. Liposomal drug formulations in cancer therapy: 15 years along the road. Drug Discov Today2012;17(3-4):160-166.

44. Yarmolenko PS, Zhao Y, Landon C et al. Comparative effects of thermosensitive doxorubicin-containing liposomes and hyperthermia in human and murine tumors. Int J Hyperthermia 2010;26(5):485-498.

45. Forssen EA, Ross ME. Daunoxome treatment of solid tumours: preclinical and clinical investigations. J Liposome Res1994;4(1):481512

46. Forssen EA, Coulter DM, Proffitt RT. Selective in vivo localization of daunorubicin small unilamellar vesicles in solid tumors. Cancer Res1992;52(12):3255-3261.

47. Lowis S, Lewis I, Elsworth A, et al. A phase 1 study of intravenous liposomal daunorubicin (daunoxome) in pediatric patients with relapsed or resistant solid tumors. Br J Cancer2006;95(5):571-580.

48. GillPS,EspinaBM,MuggiaFetal.Clinicaland pharmacokineticevaluation of liposomal Daunorubicin. J Clin Oncol1995;13(4):996-1003.

49.Guarneri V, Dieci MV, Conte P. Enhancing intracellular taxane delivery: current role and perspectives of nanoparticle albumin bound paclitaxel in the treatment of advanced breast cancer. Expert Opin Pharmacother2012;13(3):395-406.

50.Von Hoff DD, Ramanathan R, Borad M, et al. Gemcitabine plus nabpaclitaxel is an active regimen in patients with advanced pancreatic cancer: a phase I/II trial. J Clin Oncol2011;29(34):4548-54.

51. Desai N, Teieu V, Yao Z, et al. Increased antitumor activity, intratumor paclitaxel concentrations, and endothelial cell transport of cremophorfree, albumin-bound paclitaxel, ABI-007, compared with cremophorbased paclitaxel. Clin Cancer Res2006;12(4):1317-1324.

52. Desai N, Trieu V, Yao R, et al. Increased endothelial transcytosis of nanoparticle albumin-bound paclitaxel (ABI-007) by endothelial gp60 receptors: a pathway inhibited by Taxol. Proc SABCS2004;1071.

53. Gradishar WJ. Albumin-bound paclitaxel: A next-generation taxane. Expert Opin Pharmacother2006;7(8):1041-1053.

54. Sabatini P, Sill MW, O'Malley D, Adler I, Secord AA. A phase 2 trial of paclitaxel polyglumex in recurrent or persistent ovarian or primary peritoneal cancer: A Gynecologic Oncologic Group study. Gynecol Oncol2008;111(3):455-60.

55. Singer JW. Paclitaxel poliglumex (XYOTAX ${ }^{\mathrm{TM}}, \quad$ CT-2103): A macromolecular taxane. J Control Release2005;109(1-3):120-126.

56. Chipman SD, Oldham FB, Gabriella Pezzoni G, Singer JW. Biological and clinical characterization of paclitaxel poliglumex (PPX, CT-2103), a macromolecular polymer- drug conjugate. Int J Nanomedicine2006;1(4):375-383.

57. Shaffer SA, Baker-Lee C, Kennedy J, et al. In vitro and in vivo metabolism of paclitaxel poliglumex: identification of metabolites and active proteases. Cancer Chemother Pharmacol2007;59(4):537-48.

58. Betty YS Kim, James T Rutka, Warren CW. Nanomedicine. N Engl J Med2010; 363:2434-43.

59. Eichhorn ME, Ischenko I, Luedemann S et al. Vascular targeting by endotag-1 enhances therapeutic efficacy of conventional chemotherapy in lung and pancreatic cancer. Int J Cancer2010;1:126(5):1235-45.

60. Strathopoulos GP, Boulukias T. Lipoplatin formulation, review. J Drug Deliv 2012.

61. Boulikas T, Stathopoulos GP, Volakakis N, Vougiouka M. Systemic Lipoplatin infusion results in preferential tumor uptake in human studies. Anticancer Res2005;25(4):3031-3039.

62. Boulikias T. Clinical overview of Lipoplatin, a successful liposomal formulation of cisplatin. Expert Opin Investig Drugs2009;18(8):1197-218.

63. Mayer LD, Bally MB, Loughrey H, Masin D, Cullis PR. Liposomal vincristine preparations which exhibit decreased drug toxicity and increased activity against murine LI 210 and P 388 tumors. Cancer Res1990;50(3):575-579.

64.Igor VZ, Norbert M, Quet-Fah A et al. Liposome-encapsulated vincristine, vinblastine and vinorelbine: A comparative study of drug loading and retention. J Control Release2005;104(1):103-111.

65. Shih-Hung Yang, Chia Chi Lin, Zoong-Zhi Lin, Tseng YL, Hong RL. A phase 1 pharmacokinetic study of liposomal vinorelbine in patients with advanced solid tumor. Invest New drugs2012;30(1):282-289. 
66. Sankaram Mantripragada. A lipid based depot (depofoam ${ }^{\circledR}$ technology) for sustained release drug delivery. Prog Lipid Res2002;41(5):392406.

67. Angst MS, Drover DR. Pharmacology of drugs formulated with depofoam: a sustained release drug delivery system for parenteral administration using multivesicular liposome technology. Clin Pharmacokinet2006;45(12):1153-76.

68. Benesch M, Urban C. Liposomal cytarabine for leukemic and lymphomatous meningitis: recent developments. Expert Opin. Pharmacother2008;9(2):301-9.

69. Chamberlain MC, Kormanik P, Howell SB, Kim S. Pharmacokinetics of intralumbar DTC-101 for the treatment of leptomeningeal metastases. Arch Neurol1995;52(9):912-917.

70. Kim S, Chatelut E, Kim JC, et al. Extended CSF cytarabine exposure following intrathecal administration of DTC 101. J Clin Oncol1993;11(11):2186-93.

71. Pearce HL, Winter MA, Beck WT. Structural characteristics of compounds that modulate p-glycoprotein-associated multidrug resistance. Adv Enzyme Regul1990;30:357-73.

72. Boozer DJ, Esteva FJ, Rivera E, et al. Phase II study of annamycin in the treatment of doxorubicin resistant breast cancer. Cancer Chemother Pharmacol2002;50(1):6-8.

73. Immordino ML, Dosio F, Cattel L. Stealth liposomes: review of the basic science, rationale and clinical applications. Existing and potential. Int.J Nanomedicine2006;1(3):297-315.

74. Alfieri DR. Pegaspargase. Pediatr Nurs1995;21(5):471-490.

75. Avramis VI, Tiwari PN. Asparaginase (native asnase or pegylated asnase) in the treatment of acute lymphoblastic leukemia. Int $J$ Nanomedicine2006;1(3):241-254.

76. Zeidan A, Wang ES, Wetzler M. Pegasparaginase: where do we stand?. Expert Opin Biol Ther2009;9(1):111-9.

77. Emerson DL, Bendele R, Brown E et al. Antitumor efficacy, pharmacokinetics, and biodistribution of NX 211: a low clearance liposomal formulation of lurtotecan. Clin Cancer Res2000;6(7):290312

78. Dark GG, Calvert AH, Grimshaw R et al. Randomized trial of two intravenous schedules of the topoisomerase inhibitor liposomal lurtotecan in women with relapsed epithelial ovarian cancer: a trial of the national cancer institute of Canada clinical trials group. J Clin Oncol2005;23(9):1859-66.

79. Saltz LB, Cox JV, Blanke C, et al. Irinotecan plus fluorouracil and leucovorin for metastatic colorectal cancer. N Engl J Med2000;343:905-
914.

80. Douillard JY, Cunningham D, Roth AD, et al. Irinotecan combined with fluorouracil compared with fluorouracil alone as first-line treatment for metastatic colorectal cancer: A multicentre randomised trial. Lancet2000;355:1041-1047.

81. Nastruzzi C, Walde P, Menegatti E, Gambari R. Liposome-associated retinoic acid. Increased in vitro antiproliferative effects on neoplastic cells. FEBS Lett1990;259(2):293-296.

82. Parthasarathy R, Sacks PG, Harris D, Brock H, Mehta K. Interaction of liposome-associated all-trans-retinoic acid with squamous carcinoma cells. Cancer Chemother Pharmacol1994;34(6):527-534.

83. Ozpolat B, Lopez-Berestein G, Adamson P, Fu CJ, Williams AH. Pharmacokinetics of intravenously administered liposomal alltrans-retinoic acid (ATRA) and orally administered ATRA in healthy volunteers. J Pharm Sci2003;6(2):292-301.

84. Shawo J, Griffin RJ, Galanzha EI, et al. Photothermal Nanodrugs: Potential of TNF- gold nanospheres for cancer theranostics. Sci Rep2013;3:1293.

85. Day ES, Zhang L, Thompson PA, et al. Vascular-targeted photothermal therapy of an orthotopic murine glioma model. Nanomedicine (Lond)2012;7(8):1133-48.

86. Morton JG, Day ES, Halas NJ, West JL. Nanoshells for photothermal cancer therapy. Methods Mol Biol2010;624:101-17.

87. Schwartz JA., Shetty AM., Price RE et al. Feasibility study of particleassisted laser ablation of brain tumors in orthotopic canine model. Cancer Res2009;69(4):1659-1667.

88. Stern JM, Stanfield J, Kabbani W, Hsieh JT, Cadeddu JA. Selective prostate cancer thermal ablation with laser activated gold nanoshells. J Urol2008;179(2):748-53.

89. O’Neal DP, Hirsch LR, Halas NJ, Payne JD, West JL. Photo-thermal tumor ablation in mice using near infrared-absorbing nanoparticles. Cancer Lett2004;209(2):171-6.

90.Guo P. The emerging field of RNA nanotechnology. Nat Nanotechnol2010;5(12):833-42.

91. Guo S, Tschammer N, Mohammed S, Guo P. Specific delivery of therapeutic RNA to cancer clls via the dimerization mechanism of phi29 motor RNA. Hum Gen Ther2005;16(9):1097-1109.

92. Lee JM, Yoon TJ and Cho YS. Recent developments in nanoparticlebased sirna delivery for cancer therapy. Biomed Res Int2013;213:1-10.

93. Yu Kyoung Oh, Tae Gwan Park. Sirna delivery systems for cancer treatment. Advanced Drug Delivery Reviews2009;61(10):850-862. 